sentlicher Bedeutung für das ganze Problem. - Ergänzend sei vom Ref. hinzugefügt, daß nach seinen eigenen Versuchen in einem $140 \mathrm{cbm}$ groBen Laboratoriumsraum die freilebende Mehlmottenschlupfwespe Nem. ebensogut die Eph.- wie Mel.-Kulturen anflog und parasitierte. Auch ist Ref. der Ansicht, daß Nem. in ihrer Heimat (Mittel- und Ostenropa) noch mehr Wirte hat, als nur Ephestia [vgl. HASE, Arb. morph. u. taxon. Entomol. aus Bln.-Dahlem 4, 47-6x (1937)].

Ein neues Beispiel biologischer Schädlingsbekämpfung.

Der durchschlagende Erfolg der sog. biologischen Bekämpfung ${ }^{1}$ ) der Orangenschildlaus Icerya mit Hilfe des Käfers Novius cardinalis, ist genugsam beschrieben worden, gleichsam als Musterbeispiel dieses Verfahrens überhaupt. Ähnliche Erfolge biologischer Bekämpfung werden jetzt aus Australien bekannt, wo es endlich gelungen ist, die Kakteen-(Opuntien-)Plage mit Hilfe verschiedener Insekten erfolgreich $z u$ bekämpfen. Nach den letzten Nachrichten ist die Einführung. und Vermehrung des Schmetterlings Cactoblastis cactorum, von besonders gutem Erfolg gewesen. Aber auch die Blattwanze Chelinidae iabulata und die Schildlaus Dactylopius tomentosus haben sich, je nach der zu bekämpfenden Kakteenart, als brauchbar erwiesen. Tausende von Quadratkilometern guten Landes sind durch Anwendung dieses Bekämpfungsverfahrens für die Kultur gleichsam zurückerobert worden. - Auch eine andere Unkrantplage Australiens - die Überwucherung weiter Landstriche durch das eingeschleppte Johanniskraut (Hypericum perforatum) - versucht man mit Hilfe von Insekten, insbesondere durch den Käfer Chrysomela hyperià Forst., zu bekämpfen.

Wo in den tropischen und subtropischen Ländern Großkulturen von Schädlingen bedroht werden, versucht man immer wieder dieses Verfahren in Anwen. dung $z u$ bringen, vorausgesetzt, daß die allgemeinen ökologischen Verhältnisse Erfolg versprechen.

Über einen erfolgversprechenden neuen Versuch dieser Art in Columbien berichtet Murnllo in seiner kürzlich erschienenen Monographie [Sentido de una lucha biologica (Die Bedeutung einer biologischen Bekämpfung). Rev. Acad. colomb. Ci. axact. etc. I. 376-410 (I937)]. Die Baumwollpflanzungen Columbiens werden durch den , roten Kapselwurm" - es ist die Raupe des Schmetterlings Sacadodes pyral h́s DYAR aufs schwerste bedroht. Der Falter legt seine Eier an Blüte und wachsende Baumwollkapsel, wobei die Blütennektarien besonders anlockend für den Falter wirken. Durch den Raupenfraß wird die Kapsel ganz oder teilweise zerstört, was natürlich mit starkem Ernteausfall verbunden ist. MurnILo ging bei seinen Versuchen von Feldbeobachtungen aus. Er fand näm. lich in Gegenden, wo der Baumwollbau noch ziemlich primitiv ist, wenig Schädlinge, während sie in Monokulturen verheerend auftraten. Die Nachprüfung ergab, da $B$ bei primitivem Kulturbetrieb natürliche Feinde, in erster Linie eine Schlupfwespe (Braconide), die Massenvermehrung des Falters verhindern können, da sie infolge des Kulturbetriebes erhalten bleiben. Der

1) Es wäre richtiger, an Stelle von ,biologischer" Bekämpfung von ",ökologischer" Bekämpfung zu sprechen, da man bei diesem Verfahren beabsichtigt, in irgendeiner Weise die ökologischen. Verhältnisse nach einer bestimmten, von uns erwünschten Seite hin zu verschieben.
Fall wurde zum Gegenstand einer eingehenden Untersuchung gemacht, mit der Fragestellung, ob diese Braconide (die Bestimmung ergab, daß es sich um Apanteles thurberiae MuEs. handelt) zur biologischen Bekämpfung des Kapselwurmes Sacadodes bei entsprechender Massenvermehrung geeignet ist. Dies ist das Fall, da die Vermehrungspotentiale beider Formen für dieses Verfahren entsprechend geeignet sind. Die Schlupfwespe hat in 90 Tagen 3-6 Generationen (d. h. in $x_{5}$ Tagen ist eine Generation durchlaufen), während der Falter in 90 Tagen nur 2 Generationen hat. Günstig ist ferner, daß die Wespe immer nur eine Jung. raupe kurz nach dem Einbohren ansticht und dann ein neves Opfer sucht. Die parasitierte Raupe friBt nach dem Anstechen kaum noch, jedenfalls nur oberflächlich, ohne nennenswerten Schaden anzurichten. Ein entsprechend durchgeführter Feldversuch fiel durchaus günstig aus. Es wurden 90\% des Schädlings in rund I 4 Tagen durch A panteles parasitiert, und damit auf der behandelten Fläche die Ernte gerettet. Bei entsprechender praktischer Ausarbeitung verspricht demnach diese biologische Bekämpfung für Columbien von großer Wichtigkeit zu werden, da der genannte Baumwollschädling immer bedrohlicher auftritt.

\section{Blattlausmelkende Fliegen.}

Daß viele Ameisen gewisse Blattläuse teils planmäBig züchten, teils frei aufsuchen, um nach Betrillerung die ausgeschiedenen Sekrettropfen der Läuse aufzulecken, ist genugsam bekannt. Wiederholt beobachtete Herzig, wie er im Zool. Anz, i2r, H. I8/20 (I938), mitteilt, daß auch bestimmte, kleine Fliegen aus der Familie Milichiidae und Chamaemyiidae verschiedene Blattläuse in dieser Weise "melken". Interessant ist auch die weitere, gleichzeitige Beobachtung des Verf., daß an den Blattläusen bereits melkende Ameisen diese Nahrungskonkurrenten zu fangen versuchten, was aber nicht gelang. In Biologenkreisen ist dieses Verhalten einheimischer Fliegen merkwürdigerweise so gut wie unbekannt, obgleich schon 1856 der amerikanische Entomologe Fitsck und I9I2 Chr. ERvst [Biol. Zbl. 32, I55 (rgi2)] und I928 der Engländer MrCHEL MORE [Entomologist (Lond.) 6I, 90-9I ( $\mathrm{g} 28)$ darauf aufmerksam machten. Thre Beobachtungen bezogen sich aber zum Teil auf Bohrfliegen (Trypetidae) und auf Musciden (Fanniaarten). Demnach scheint diese Art der Nahrungsgewinnung unter den Fliegen weiter verbreitet zu sein.

Albrecht Hase.

\section{Berichtigung.}

In unserer Mitteilung: ,Uber die isomeren 4,4'-Dioxy- $\gamma, \delta$ diphenyl $* n$-hexene: [Naturwiss. 27,567-568(1939)] soll es auf Seite 567 in der Tabelle, in der die biologische Wirksamkeit angegeben ist, heiBen: statt $4,4^{\prime}$-Dioxy $\mu, \delta$-diphenyl- $\beta$-hexan $4,4^{\prime}-$ Dioxy $-\gamma, \delta$-diphenyl- $\beta$-hexen und statt $4,4^{\prime}-$ Dioxy- $\gamma, \delta$ diphenyl-n-hexen $4,4^{\prime}$-Dioxy $\gamma$, $\delta$-diphenyl-n-hexan.

Wien, den Ir. September r 939 . II. Chemisches Universitätslaboratorium. F. v. WESSELY, A. KLEEDORFER.

\section{Berichtigung.}

In der kurzen Originalmitteilung von $\mathrm{H}$. Kiessig und W. PhILIppofs , Rönigenographische und viskosimetrische Untersuchungen über die Wirkung von Zusätzen auf den Aufbau von Natriumoleatlösungens" in Heft $35, \mathrm{~S} .593 \mathrm{muB}$ es S. 594 , linke Spalte, 8 . Zeile von oben statt Ir $3 \AA^{1}$ heißen: Ir $3 \AA^{3}$. Die Fußnoten $x$ und 2 auf dieser Spalte sind $z u$ streichen. Es liegt ein Versehen des Setzers vor.

Die Redaktion. 